\title{
A large strain anisotropic elastoplastic continuum theory for nonlinear kinematic hardening and texture evolution
}

\author{
Francisco Javier Montáns ${ }^{a}, *$, José María Benítez ${ }^{a}$, Miguel Ángel Caminero ${ }^{b}$ \\ ${ }^{a}$ Escuela Técnica Superior de Ingenieros Aeronáuticos, Universidad Politécnica de Madrid, Pza. Cardenal Cisneros 3, 28040 Madrid, Spain \\ ${ }^{\mathrm{b}}$ Escuela Técnica Superior de Ingenieros Industriales, Universidad de Castilla-La Mancha, Campus Universitario s/n, 13071 Ciudad Real, Spain
}

Keywords:

Large strains

Logarithmic strains

Hyperelasticity

Texture evolution

Nonlinear kinematic hardening

\begin{abstract}
A B S T R A C T
In this paper we present a continuum theory for large strain anisotropic elastoplasticity based on a decomposition of the modified plastic velocity gradient into energetic and dissipative parts. The theory includes the Armstrong and Frederick hardening rule as well as multilayer models as special cases even for large strain anisotropic elastoplasticity. Texture evolution may also be modelled by the formulation, which allows for a meaningful interpretation of the terms of the dissipation equation.
\end{abstract}

\section{Introduction}

Efficiency and accuracy of finite element simulations of elastoplastic behaviour are very important because much of the computational cost is due to the stress point integration algorithms and the predicted behaviour depends completely on the material constitutive relations. Current algorithms widely used in industry are based on continuum mechanics assumptions, in which some simplifications are made in order to obtain an engineering solution (Bathe, 1996; Kojić and Bathe, 2005). Many large strain simulations of metal sheet goods are performed using isotropic elastic relations with isotropic or anisotropic yield functions, using algorithms derived from the works of Weber and Anand (1990), Eterovic and Bathe (1990) and Simó (1992), among others, which use hyperelastic relations, objective stress integration algorithms and a simple modular structure in which large strain kinematics reduce to a geometric pre- and post-processor. Several formulations have been proposed for anisotropic elastoplasticity, but those do not inherit the modular structure and use plastic metrics, Green additive decompositions of total strains or elastic isotropy, see for example (Papadopoulos and Lu, 2001; Miehe et al., 2002; Menzel and Steinmann, 2003; Ulz, 2009; Vladimirov et al., 2010), among others. Additive splits of total (versus incremental) strains into elastic and plastic parts have been questioned in Ref. Schmidt (2005) regard-

\footnotetext{
* Corresponding author.

E-mail addresses: fco.montans@upm.es (F.J. Montáns), josemaria.benitez@upm.es (J.M. Benítez), miguelangel.caminero@uclm.es (M.Á. Caminero).
}

ing the resulting unexpected constitutive equations. Furthermore, in those formulations the plastic spin is usually assumed to vanish and the effects of texture evolution with strain are not taken into account, even when it has been experimentally observed (Kim and Yin, 1997; Truong and Lippmann, 2001; Boheler and Koss, 1991). On the other hand, the physical meaning of a nonvanishing continuum plastic spin has been studied in many works (see for example (Loret, 1983; Dafalias, 1985, 1998; Kuroda and Tvergaard, 2001; Tong et al., 2004) and therein references), some related to texture evolution but not motivated from an energetic contribution. Instead ad hoc constitutive expressions are used, as for example in Choi et al. (2006a,b). Furthermore, these formulations do not frequently include mixed hardening, specially nonlinear kinematic hardening as given by the Armstrong and Frederick rule, which in small strains is usually modelled as a type of nonassociative hardening, see for example (Lemaitre and Chaboche, 1990).

In this paper we present a novel view of anisotropic elastoplasticity in which both texture evolution and nonlinear kinematic hardening are considered at large strains in an associative manner. Multilayer models (Montáns, 2001, 2004; Montáns and Caminero, 2007) may also be included in the framework in a straightforward manner. The theory uses the Lee decomposition of the deformation gradient into elastic and plastic parts. The resulting (modified) plastic velocity gradient, plastic deformation rate and plastic spin tensors are then divided into energetic and dissipative contributions. The formulation is established from a decomposition similar to (but different from) that of Lion (2000) which was used recently by Hennan and Anand (2009) and Vladimirov et al. (2010) to model the Armstrong and Frederick rule in isotropic elastoplasticity and anisotropic elastoplasticity respectively, see also therein 
references. However, our formulation, for which we justify our preference, allows for a simpler interpretation and procedure. Furthermore, including the plastic spin effects allows us to model texture evolution in anisotropic elastoplasticity motivated from the dissipation equation and from the energetic contribution. Then, the formulation given in Montáns and Bathe (2007) for texture evolution (used to predict the Kim and Yin experiments (Kim and Yin, 1997)) is obtained in a parallel way to that of the Armstrong and Frederick rule and a clear physical interpretation is obtained for the different spin contributions of Montáns and Bathe (2007).

Finally, the theory is developed using logarithmic strains which allows for an intuitive interpretation of the formulation. If a quadratic stored energy function in terms of logarithmic strains and an exponential mapping are used, a simple modular structure (thanks to the additive decomposition of incremental strains) is obtained in which the plastic strain integration is performed using a small strains procedure. The large strain kinematics reduce to a pre- and post-processor. Details of a numerical implementation of this type for anisotropic elasto-plasticity may be found in Montáns and Bathe (2005) and Caminero et al. (2011), remaining basically unaltered except for the use of a proper small strain procedure which includes the Armstrong and Frederick rule (or a multilayer model). The integration of the skew-symmetric part may be performed uncoupled from that of the symmetric part (see details in Montáns and Bathe (2007)). Thus, we refer to those references for examples and numerical details which are essentially valid in the present theory.

\section{Motivation: small strain plasticity model}

\subsection{The principle of maximum dissipation}

The stress power per unit volume for small displacements may be written as

$\mathcal{P}=\sigma: \nabla v=\sigma:(\dot{\varepsilon}+\dot{\omega})=\sigma: \dot{\varepsilon}=\sigma: \dot{\varepsilon}^{e}+\sigma: \dot{\varepsilon}^{p}$

where $v$ is the velocity and $\nabla v$ is the velocity gradient, $\dot{\varepsilon}$ is the small strain rate tensor and $\dot{\omega}$ is the skew-symmetric infinitesimal spin tensor, which produces no power. As usual, we assumed an additive decomposition of the total small strain tensor rate into an elastic and a plastic part $\dot{\varepsilon}=\dot{\varepsilon}^{e}+\dot{\boldsymbol{\varepsilon}}^{p}$ so the plastic strain rate is obtained from the constitutive equation in rate form, according to the path-dependent nature of plastic strains.

As mentioned, an arguably intuitive, general and elegant theory due to Lion (2000) has been used recently in the context of large strains in order to extend the Armstrong and Frederick hardening rule to large strains. The basic idea of this theory is to further divide the plastic contribution into an energetic and a dissipative part, which in small strains may be modelled using an additive split of the plastic strain rate $\dot{\varepsilon}^{p}=\dot{\varepsilon}^{p e}+\dot{\varepsilon}^{p d}$. Both $\varepsilon^{p}$ and $\varepsilon^{p d}$ are obtained through integration of their rates, which are given by the proper constitutive equations in rate form. The split of the plastic strains is equivalent to the traditional split into plastic kinematic strains contribution and plastic isotropic strains contribution, which is the basis of the split of plastic strains (SPS) method (Bathe and Montáns, 2004) to model mixed hardening. Then, the internal power is enlarged with two terms which cancel out each other

$\mathcal{P}=\sigma: \dot{\boldsymbol{\varepsilon}}^{e}+\sigma: \dot{\boldsymbol{\varepsilon}}^{p}=\sigma: \dot{\boldsymbol{\varepsilon}}^{e}+\sigma: \dot{\boldsymbol{\varepsilon}}^{p}+\underbrace{\boldsymbol{s}^{p e}: \dot{\boldsymbol{\varepsilon}}^{p e}+\boldsymbol{s}^{p d}: \dot{\boldsymbol{\varepsilon}}^{p d}}_{=0}$

where $\boldsymbol{s}^{p e}$ and $\boldsymbol{s}^{p d}$ are adequate stress-like internal variables. The free energy is of the form $\psi=\mathcal{W}\left(\boldsymbol{\varepsilon}^{e}\right)+\mathcal{H}\left(\boldsymbol{\varepsilon}^{p e}\right)$; the first contribution is due to the elastic strains which are recovered if the continuum stresses are locally released, whereas the second contribution accounts for elastic strains blocked in the system by successive plastic slips and which can be recovered only with local plastic flow along certain routes (as in a sliding puzzle). One can think of it as modelling two different scales, $\mathcal{W}\left(\boldsymbol{\varepsilon}^{e}\right)$ being the energy at a macroscopic scale (due to continuum stresses and strains) and $\mathcal{H}\left(\boldsymbol{\varepsilon}^{\text {pe }}\right)$ being the energy at a microscopic scale (not considered in the Cauchy stresses and strains, but later accounted for through a continuum stress measure: the backstress tensor), see Vladimirov et al. (2010). Then, the dissipation equation takes the form

$$
\begin{aligned}
\mathcal{D} & =\mathcal{P}-\dot{\psi}=\left(\sigma-\frac{\partial \mathcal{W}}{\partial \boldsymbol{\varepsilon}^{e}}\right): \dot{\boldsymbol{\varepsilon}}^{e}+\sigma: \dot{\boldsymbol{\varepsilon}}^{p}+\left(\boldsymbol{s}^{p e}-\frac{\partial \mathcal{H}}{\partial \boldsymbol{\varepsilon}^{p e}}\right): \dot{\boldsymbol{\varepsilon}}^{p e} \\
& +\boldsymbol{s}^{p d}: \dot{\boldsymbol{\varepsilon}}^{p d} \geq 0
\end{aligned}
$$

so since the equality holds for purely elastic responses $\sigma=\partial \mathcal{W} / \partial \boldsymbol{\varepsilon}^{e}$. During plastic flow there is an additional part of the internal energy which is not dissipated taking (these are the backstresses)

$\mathbf{s}^{p e}=\frac{\partial \mathcal{H}}{\partial \boldsymbol{\varepsilon}^{p e}}$

The plastic dissipation is now

$\mathcal{D}^{p}=\sigma: \dot{\boldsymbol{\varepsilon}}^{p}+\boldsymbol{s}^{p d}: \dot{\boldsymbol{\varepsilon}}^{p d}=\sigma: \dot{\boldsymbol{\varepsilon}}^{p}-\boldsymbol{s}^{p e}: \dot{\boldsymbol{\varepsilon}}^{p e} \geq 0$

If one considers the yield functions $f_{y}\left(\sigma-\boldsymbol{s}^{p e}, \kappa\right)$ and $g_{y}\left(\boldsymbol{s}^{p e}, \kappa^{g}\right)$, which may be anisotropic, where $\kappa$ and $\kappa^{g}$ are material parameters, then the Lagrangean

$\mathcal{L}=\sigma: \dot{\boldsymbol{\varepsilon}}^{p}-\mathbf{s}^{p e}: \dot{\boldsymbol{\varepsilon}}^{p e}-\dot{t} f_{y}-\dot{\gamma} g_{y}$

yields the constrained maximum condition

$\nabla \mathcal{L}=0 \Rightarrow\left\{\begin{array}{l}\dot{\boldsymbol{\varepsilon}}^{p}=\dot{t} \partial f_{y} / \partial \boldsymbol{\sigma} \\ \dot{\boldsymbol{\varepsilon}}^{p e}=-\dot{t} \partial f_{y} / \partial \mathbf{s}^{p e}-\dot{\gamma} \partial g_{y} / \partial \boldsymbol{s}^{p e}=\dot{t} \partial f_{y} / \partial \boldsymbol{\sigma}-\dot{\gamma} \partial g_{y} / \partial \mathbf{s}^{p e}=\dot{\boldsymbol{\varepsilon}}^{p}-\dot{\boldsymbol{\varepsilon}}^{p d}\end{array}\right.$

The meaning of $g_{y}$ for the backstresses is similar to that of $f_{y}$ for the stresses and for a limit on $\psi$. Obviously an isotropic hardening may be added to the theory with no difficulty through a derivative $\dot{\kappa}$ which may be function of $\dot{\boldsymbol{\varepsilon}}^{p d}$. For brevity we refer to the discussion on the nature of this hardening (which may be related to a defect energy) given in Gurtin et al. (2010) and in Hennan and Anand (2009), see also Vladimirov et al. (2010) for a different but more usual approach.

\subsection{The Armstrong-Frederick nonlinear kinematic hardening rule}

Usually, the Armstrong-Frederick nonlinear kinematic hardening rule is introduced phenomenologically as nonassociative hardening in order to introduce a dynamic recall term, see for example Lemaitre and Chaboche (1990). With the above setting, one may simply assume a potential function $g_{y}\left(\mathbf{s}^{p e}\right)=1 / 2\left\|\mathbf{s}^{p e}\right\|^{2}$. Then $\partial g_{y} / \partial \mathbf{s}^{p e}=\mathbf{s}^{p e}$, and

$\dot{\boldsymbol{\varepsilon}}^{p e}=\dot{t} \partial f_{y} / \partial \boldsymbol{\sigma}-\dot{\gamma} \mathbf{s}^{p e}$

In order to obtain the familiar hardening description assume

$\mathcal{H}=\frac{1}{3} H\left\|\boldsymbol{\varepsilon}^{p e}\right\|^{2}$ so $\frac{\partial \mathcal{H}}{\partial \boldsymbol{\varepsilon}^{p e}}=\frac{2}{3} H \boldsymbol{\varepsilon}^{p e}$

Then by Eqs. (4) and (7), using a constant hardening parameter $H$

$\dot{\boldsymbol{s}}^{p e}=\frac{2}{3} H \dot{\boldsymbol{\varepsilon}}^{p e}=\frac{2}{3} H \dot{t} \frac{\partial f_{y}}{\partial \boldsymbol{\sigma}}-\dot{\gamma} \frac{2}{3} H \boldsymbol{s}^{p e}$

Finally, one may set a linear relationship between both consistency parameters through a material parameter $b$ in a conceptually similar way as in viscoplasticity (this relationship establishes the amount of plastic strain that is energetic):

$\dot{\gamma}=\frac{b}{2 / 3 H} \dot{t}$ 
Thus, the usual expression for the Armstrong and Frederick rule is obtained as a special case for this formulation:

$\dot{\boldsymbol{s}}^{p e}=\dot{t}\left(\frac{2}{3} H \frac{\partial f_{y}}{\partial \sigma}-b \boldsymbol{s}^{p e}\right)$

In Hennan and Anand (2009) a viscoplastic-like relation is also used to accommodate an Armstrong-Frederick type of kinematic hardening at large strains, but their work is not derived from an specific potential function and Eq. (7). In Vladimirov et al. (2010) the same consistency parameter is used for plastic and plastic inelastic deformation rates, resulting in an equivalent expression to Eq. (11). In this work $g_{y}$ accounts for the level that $s^{p e}$ (stresses at the microstructure (Vladimirov et al., 2010)) achieve.

\section{Large strain anisotropic plasticity formulation which accounts for nonlinear kinematic hardening and texture evolution}

In this section we extend the previous concepts to large strain elastoplasticity, allowing also for texture evolution. As mentioned, the formulation is designed such that the attractive structure of the computational algorithms for isotropic elasto-plasticity is kept. The goal of the present paper is to show that using similar concepts, two effects may be modelled at large strains. The first one is the Armstrong and Frederick type of kinematic hardening (and many other as nonlinear kinematic models) without restriction to isotropy. The second one is the texture evolution, present in anisotropic elastoplastic metals and experimentally observed for large strains. In particular, a remarkable novelty of the continuum formulation given below is the inclusion of the possible texture evolution modelled through an energetic spin which subtract to the plastic spin in the dissipation equation, so the dissipative spin (the difference between both spins) is given by the principle of maximum dissipation through a proper potential function for a skew-symmetric Cosserat-like stress tensor. The framework is then parallel to that given for the Armstrong and Frederick rule.

\subsection{Multiplicative decomposition and strain rate tensors}

The notation used in this section follows closely the notation of References (Bathe, 1996) and (Kojić and Bathe, 2005). The Lee multiplicative decomposition provides the following decomposition of the deformation gradient

${ }_{0}^{t} \boldsymbol{X}={ }_{0}^{t} \boldsymbol{X}_{0}^{e t} \boldsymbol{X}^{p}$

The intermediate configuration is defined by a local elastic unload ${ }_{0}^{t} \boldsymbol{X}^{p}=\left({ }_{0}^{t} \boldsymbol{X}^{e}\right)^{-1}{ }_{0}^{t} \boldsymbol{X}$, where ${ }_{0}^{t} \boldsymbol{X}^{e}$ also contains any superimposed rigid body motion. Hence ${ }_{0}^{t} \boldsymbol{X}^{p}$ is invariant under arbitrary rigid body motions. As noted in Refs. (Hennan and Anand, 2009) and (Gurtin et al., 2010), the invariance principle does not apply to the intermediate configuration (at least in anisotropy). Aside, the intermediate configuration is uniquely obtained through "time" integration of the constitutive equations. Both $\boldsymbol{X}^{e}$ and $\boldsymbol{X}^{p}$ are incompatible, but $\boldsymbol{X}^{e}$ is such that $\boldsymbol{X}$ is a compatible deformation at a continuum level. We can define the modified velocity gradient ${ }^{t} \boldsymbol{L}$ as the pull-back to the intermediate configuration of the spatial velocity gradient

${ }^{t} \boldsymbol{L}={ }_{0}^{t} \boldsymbol{X}^{e T t}{ }_{0}^{e} \dot{\boldsymbol{X}}^{e}+{ }_{0}^{t} \boldsymbol{X}^{e^{T}}{ }_{0}^{t} \boldsymbol{X}^{e t} \boldsymbol{L}^{p}={ }^{t} \boldsymbol{L}^{e}+{ }^{t} \boldsymbol{C}^{e t} \boldsymbol{L}^{p}$

where ${ }^{t} \boldsymbol{C}^{e} \equiv{ }_{0}^{t} \boldsymbol{X}^{e^{T}}{ }_{0}^{t} \boldsymbol{X}^{e}$ is the right Green-Cauchy elastic deformation tensor in the intermediate configuration, ${ }^{t} \boldsymbol{L}^{e}:={ }_{0}^{t} \boldsymbol{X}^{e T}{ }_{0}^{t} \dot{\boldsymbol{X}}^{e}$ and

${ }^{t} \boldsymbol{L}^{p}:={ }_{0}^{t} \dot{\boldsymbol{X}}^{p_{t}} \boldsymbol{X}^{p-1}$ is the modified plastic velocity gradient in the same configuration. From the Polar Decomposition Theorems of the elastic deformation gradient tensor,

$\boldsymbol{X}^{e}=\boldsymbol{R}^{e} \boldsymbol{U}^{e}=\boldsymbol{V}^{e} \boldsymbol{R}^{e}$

where $\boldsymbol{R}^{e}$ is the "elastic" rotation which includes the rigid body motion and $\boldsymbol{U}^{e}$ and $\mathbf{V}^{e}$ are the stretch tensors from which the Seth-Hill elastic strain measures may be defined. In elastic (recoverable) deformations the Polar Decomposition Theorem separate deformations and rotations. The logarithmic strain measures are specially interesting for several reasons. First, hyperelasticity in metals may be rather accurately modelled in terms of logarithmic strains with constant coefficients (Anand and On, 1979). Second, they yield additive incremental algorithms at large strains (Montáns and Bathe, 2005). And third, in contrast with quadratic measures, the push-forward and pull-back operations are made with the rotation part alone, so the metric does not change (both for strains and for their work-conjugate stress measures), an important advantage in anisotropic plasticity (Schmidt, 2005):

$\boldsymbol{e}^{e}=\ln \boldsymbol{V}^{e}, \boldsymbol{E}^{e}=\ln \boldsymbol{U}^{e} \quad$ and $\quad \boldsymbol{e}^{e}=\boldsymbol{R}^{e} \boldsymbol{E}^{e} \boldsymbol{R}^{e T}$

On the other hand, using Eq. (15), the evolution of the plastic deformation strain gradient is given by the differential equation

${ }_{0}^{t} \dot{\boldsymbol{X}}^{p}={ }^{t} \boldsymbol{L}_{0}^{p t} \boldsymbol{X}^{p}=\left({ }^{t} \boldsymbol{D}^{p}+{ }^{t} \boldsymbol{W}^{p}\right){ }_{0}^{t} \boldsymbol{X}^{p}$

In computational elastoplasticity it is now customary to use an exponential integration (see Vladimirov et al. (2010) for a evaluation in closed form) which we will use as an aid in the following arguments. For small $\Delta t$ that update may be written as

${ }_{0}^{t+\Delta t} \boldsymbol{X}^{p} \simeq \exp \left(\Delta t^{t} \boldsymbol{W}^{p}\right) \exp \left(\Delta t^{t} \boldsymbol{D}^{p}\right){ }_{0}^{t} \boldsymbol{X}^{p}$

where

$\exp \left(\Delta t^{t} \boldsymbol{W}^{p}\right)={ }^{t+\Delta t}{ }_{t} \boldsymbol{R}^{w}$

is a rotation (orthogonal) tensor and

${ }_{t}^{t+\Delta t} \overline{\boldsymbol{U}}^{p}:=\exp \left(\Delta t^{t} \boldsymbol{D}^{p}\right)$

We now consider two consecutive steps

${ }_{0}^{2} \boldsymbol{X}^{p}={ }_{1}^{2} \boldsymbol{X}_{0}^{p 1} \boldsymbol{X}^{p} \simeq{ }_{1}^{2} \boldsymbol{R}_{1}^{w 2} \overline{\boldsymbol{U}}_{0}^{p}{ }_{0}^{1} \boldsymbol{R}_{0}^{w 1} \overline{\boldsymbol{U}}^{p}$

Obviously, a polar decomposition of the plastic deformation gradient yields

${ }_{0}^{2} \boldsymbol{X}^{p}={ }_{0}^{2} \boldsymbol{R}_{0}^{p 2} \boldsymbol{U}^{p} \quad$ with ${ }_{0}^{2} \boldsymbol{U}^{p} \neq{ }_{1}^{2} \overline{\boldsymbol{U}}_{0}^{p} \overline{\boldsymbol{U}}^{p}$ and ${ }_{0}^{2} \boldsymbol{R}^{p} \neq{ }_{1}^{2} \boldsymbol{R}_{0}^{w 1} \boldsymbol{R}^{w}$

Since plastic strains are in principle not recoverable, permanent (due to slip mechanisms in grains), new steps cannot change the rotation or deformation character of previous, occurred deformations. We obviously assumed that incremental plastic gradients are always applied to the left of previous plastic gradients. Hence, it is arguable the use of $\boldsymbol{U}^{p}$ or other total (versus incremental) strain measures in constitutive equations, specially in anisotropy (the situation is rather opposite to the elastic part). Furthermore, as seen below, the rotations derived from the plastic spin do not dissipate energy in elastically isotropic solids, whereas the plastic deformations derived from $\boldsymbol{D}^{p}$ do dissipate energy. In general, if we assume $\boldsymbol{W}^{p}=\mathbf{0}$, then two consecutive steps with principal directions not aligned yield

${ }_{0}^{2} \boldsymbol{X}^{p}={ }_{1}^{2} \overline{\boldsymbol{U}}_{0}^{p}{ }_{0}^{1} \overline{\boldsymbol{U}}^{p}=\exp \left[\left(t_{2}-t_{1}\right){ }^{1} \boldsymbol{D}^{p}\right] \exp \left[\left(t_{1}-t_{0}\right)^{0} \boldsymbol{D}^{p}\right]={ }_{0}^{2} \boldsymbol{R}_{0}^{p 2} \boldsymbol{U}^{p}$

with ${ }_{0}^{2} \boldsymbol{R}^{p} \neq \boldsymbol{I}$, so considering only the total ${ }_{0}^{2} \boldsymbol{U}^{p}$ in the constitutive equation (or any derived Seth-Hill total plastic measure) may yield an undesired plastic dissipation, an argument which adds to those given in Schmidt (2005). Thus we prefer to see plastic deformations 
as incremental, which is also the way the equivalent plastic strain is computed.

A similar reasoning (but of more complex interpretation) may be employed in the decomposition of the plastic deformation gradient into an energetic and a dissipative part, as considered in Vladimirov et al. (2010), Lion (2000), Hennan and Anand (2009)

$\boldsymbol{X}^{p}=\boldsymbol{X}^{p e} \boldsymbol{X}^{p d}$

Since plastic energetic and plastic dissipative deformations occur simultaneously due to slips in grains, two consecutive steps would give

${ }_{0}^{2} \boldsymbol{X}^{p}={ }_{1}^{2} \boldsymbol{X}_{1}^{p e 2} \boldsymbol{X}_{0}^{p d 1} \boldsymbol{X}_{0}^{p e 1} \boldsymbol{X}^{p d}$

so the energetic part may not be easily recovered with operations of the type

$\boldsymbol{X}^{p e}=\boldsymbol{X}^{p}\left(\boldsymbol{X}^{p d}\right)^{-1}$

which for us defines the difference between the plastic origin of $\boldsymbol{X}^{p e}$ and the elastic origin of $\boldsymbol{X}^{e}$. The deformations due to $\boldsymbol{X}^{e}$ are recovered in a local elastic unload (continuum), whereas in order to recover $\boldsymbol{X}^{\text {pe }}$ plastic flow is needed which somehow reverses at least part of the previous path (as in a sliding puzzle). Aside, the plastic deformations at the initial, reference configuration are usually unknown. Thus we consider an open question whether the hypothesis of decomposition (25) is the most adequate one for this case. We note that whereas $\mathbf{X}$ in (13) is a compatible deformation gradient, $\mathbf{X}^{p}$ in (25) is not necessarily a compatible deformation gradient in a continuum. Instead of decomposition (25), we propose an additive split of the (modified) plastic velocity gradients

$\boldsymbol{L}^{p}=\boldsymbol{L}^{p e}+\boldsymbol{L}^{p d}$

and similar additive decompositions into deformation rates and spin tensors

$\boldsymbol{D}^{p}=\boldsymbol{D}^{p e}+\boldsymbol{D}^{p d}$

$\boldsymbol{W}^{p}=\boldsymbol{W}^{p e}+\boldsymbol{W}^{p d}$

so

$\boldsymbol{L}^{p e}=\boldsymbol{D}^{p e}+\boldsymbol{W}^{p e}$ and $\boldsymbol{L}^{p d}=\boldsymbol{D}^{p d}+\boldsymbol{W}^{p d}$

These decompositions yield interesting results in the dissipation equation which are not as easy to interpret if we insist in using decomposition (25).

\subsection{Dissipation inequality}

In an isothermal process, the stress power $\mathcal{P}$ of external forces (in the reference volume) may be expressed in the intermediate configuration as, see Refs. (Montáns and Bathe, 2007, 2005)

$\mathcal{P}=\boldsymbol{S}: \boldsymbol{L}=\boldsymbol{S}:\left(\boldsymbol{L}^{e}+\boldsymbol{C}^{e} \boldsymbol{L}^{p}\right)=\boldsymbol{S}:\left(\boldsymbol{D}^{e}+\boldsymbol{W}^{e}\right)+\boldsymbol{S}: \boldsymbol{C}^{e}\left(\boldsymbol{D}^{p}+\boldsymbol{W}^{p}\right)$

where $\boldsymbol{S}$ is the pull-back of the Kirchhoff stress $\boldsymbol{\tau}$ to the stress-free configuration. Since $\boldsymbol{S}$ is symmetric, the product $\boldsymbol{S}: \boldsymbol{W}^{e}=0$, i.e, the elastic spin produces no work. Using the Mandel stress tensor $\boldsymbol{\Xi}:=$ $\boldsymbol{C}^{e} \boldsymbol{S}$, the stress power can be written as

$\mathcal{P}=\boldsymbol{S}: \boldsymbol{L}=\boldsymbol{S}: \boldsymbol{D}^{e}+\left(\boldsymbol{\Xi}_{s}+\boldsymbol{\Xi}_{w}\right):\left(\boldsymbol{D}^{p}+\boldsymbol{W}^{p}\right)=\boldsymbol{S}: \boldsymbol{D}^{e}+\boldsymbol{\Xi}_{s}: \boldsymbol{D}^{p}$

$+\boldsymbol{\Xi}_{w}: \boldsymbol{W}^{p}$

to which, similarly to the small strain case, we add four terms which cancel out two by two

$\mathcal{P}=\boldsymbol{S}: \boldsymbol{D}^{e}+\boldsymbol{\Xi}_{s}: \boldsymbol{D}^{p}+\boldsymbol{\Xi}_{w}: \boldsymbol{W}^{p}$

$$
+\underbrace{\boldsymbol{\beta}^{p e}: \boldsymbol{D}^{p e}+\boldsymbol{\beta}^{p d}: \boldsymbol{D}^{p d}}_{=0}+\underbrace{\boldsymbol{T}_{w}^{p e}: \boldsymbol{W}^{p e}+\boldsymbol{T}_{w}^{p d}: \boldsymbol{W}^{p d}}_{=0}
$$

The tensors $\boldsymbol{\Xi}_{s}$ and $\boldsymbol{\Xi}_{w}$ are the symmetric and skew part of the Mandel stress tensor, $\beta^{p e}, \beta^{\text {pd }}$, are symmetric stress-like tensors, and $\boldsymbol{T}_{w}^{p e}$ and $\boldsymbol{T}_{w}^{p d}$ are skew-symmetric stress-like tensors. From Eq. (34) it is seen that the symmetric part of the Mandel stress tensor $\boldsymbol{\Xi}_{S}$ produces power on the modified plastic strain rate, whereas the skew part of the Mandel stress tensor $\boldsymbol{\Xi}_{w}$ produces power on the modified plastic spin. In the case of elastic isotropy, stresses and strains commute because they have the same eigenvectors, hence $\boldsymbol{\Xi}_{w}=\mathbf{0}$ and the plastic spin produces no dissipation nor energy change, so a value of $\boldsymbol{W}^{p}=\mathbf{0}$ is a common and natural choice from an energetic point of view.

Motivated in the work of Anand for isotropic metals (Anand and On, 1979), we use for motivation and simplicity of the presentation (but without loss of the generality of the theory) the following stored energy function, which is also an attractive choice for computational implementation

$\psi=\mathcal{W}+\mathcal{H}=\frac{1}{2} \boldsymbol{E}^{e}: \mathbb{A}: \boldsymbol{E}^{e}+\frac{1}{2} \boldsymbol{E}^{p e}: \mathbb{H}: \boldsymbol{E}^{p e}$

where $\mathbb{A}$ is a tensor of elastic constants similar to those used for small strains and $\boldsymbol{E}^{e}$ is the logarithmic elastic strain tensor in the intermediate configuration and $\boldsymbol{E}^{p e}$ are energetic logarithmic strains given below. For practical reasons, we will also assume a constant hardening tensor $\mathbb{H}$ that has the same principal orthotropy directions as $\mathbb{A}$, which in essence are due to material texture.

Since push-forward and pull-back operations for logarithmic measures are performed with the rotation part and we have seen that we need to consider only the incremental rotation $\boldsymbol{R}^{w}$ in the push-forward and pull-back between intermediate configurations during stress integration, we define a configuration where the incremental plastic rotation did not take place by $(\cdot)$, hence

${ }^{t} \boldsymbol{E}^{e}=\boldsymbol{R}^{w T t} \boldsymbol{E}^{e} \boldsymbol{R}^{w}$

and we can define a Lie-type derivative for logarithmic strains and their work-conjugate stress measures in the intermediate configuration as

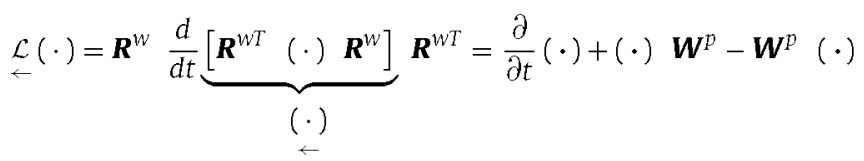

We can consider the free energy in the intermediate configuration, which at a given step is defined by ( ) , i.e.

$\psi=\frac{1}{2} \boldsymbol{E}_{\leftarrow}^{e}: \underset{\leftarrow}{\mathbb{A}}: \underset{\leftarrow}{\boldsymbol{E}^{e}}+\frac{1}{2} \boldsymbol{E}_{\leftarrow}^{p e}: \underset{\leftarrow}{\mathbb{H}}: \underset{\leftarrow}{\boldsymbol{E}^{p e}}$

The variation of the free energy with this configuration fixed (to simplify the presentation since the energy is obviously invariant) has a contribution due to the change of strains and a contribution due to texture evolution (principal directions or structural tensors evolution in general)

$\dot{\psi}=\underset{\leftarrow}{\boldsymbol{E}^{e}}: \underset{\leftarrow}{\mathbb{A}}: \dot{\boldsymbol{E}}_{\leftarrow}^{e}+\frac{1}{2} \underset{\leftarrow}{\boldsymbol{E}^{e}}: \underset{\leftarrow}{\dot{\mathbb{A}}}: \underbrace{\boldsymbol{E}^{e}}_{\leftarrow}+\underset{\leftarrow}{\boldsymbol{E}^{p e}}: \underset{\leftarrow}{\mathbb{H}}: \dot{\leftarrow}^{\dot{\boldsymbol{E}}^{p e}}+\frac{1}{2} \boldsymbol{E}_{\leftarrow}^{p e}: \underset{\leftarrow}{\dot{\mathbb{H}}}: \underbrace{\boldsymbol{E}^{p e}}$ 
The time derivatives in the anisotropy tensors correspond to the rotation of the principal anisotropy directions due to texture evolution. We define the following stress tensors

$\underset{\leftarrow}{\boldsymbol{T}}=\frac{\partial \psi}{\partial \boldsymbol{E}^{e}}=\underset{\leftarrow}{\boldsymbol{E}^{e}}: \underset{\leftarrow}{\mathbb{A}} ; \quad \underset{\leftarrow}{\boldsymbol{B}}=\frac{\partial \psi}{\partial \boldsymbol{E}^{p e}}=\underset{\leftarrow}{\boldsymbol{E}^{p e}}: \underset{\leftarrow}{\mathbb{H}} ;$

with

$\boldsymbol{T}=\boldsymbol{R}^{w} \boldsymbol{T}^{W T}$

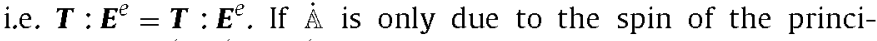
pal orthotropy directions (our simplifying hypothesis) which we denote by $\boldsymbol{W}^{A}=\dot{\boldsymbol{R}}^{A} \boldsymbol{R}^{A}{ }^{T}$, where $\boldsymbol{R}^{A}$ is the rotation of the principal directions, we can write in a configuration in which incremental plastic rotations did not take place

$\frac{1}{2} \boldsymbol{E}^{e}: \underset{\leftarrow}{\dot{\mathbb{A}}}: \boldsymbol{E}_{\leftarrow}^{e}=\underset{\leftarrow}{\boldsymbol{T}}: \boldsymbol{W}^{A}=\boldsymbol{T}_{w}: \boldsymbol{W}^{A}$

where we defined the skew-symmetric stress tensor in the updated intermediate configuration

$\boldsymbol{T}_{w}:=\boldsymbol{E}^{e} \boldsymbol{T}-\boldsymbol{T E}^{e}$

The tensor $\boldsymbol{T}_{w}$ is not an independent quantity (it only depends on $\boldsymbol{E}^{e}$ and on the elastic constants) and measures the noncoaxiality between stresses and strains, giving an enlightening interpretation of this contribution to the stress power. A similar reasoning is valid for the kinematic hardening contribution, where

$\frac{1}{2} \boldsymbol{E}^{\text {pe }}: \underset{\mathbb{H}}{\leftarrow}:{\underset{\boldsymbol{E}}{\text { pe }}}^{\mathrm{s}}=\boldsymbol{B}_{w}: \boldsymbol{W}^{H}$

with $\boldsymbol{W}^{H}$ being the spin of the principal anisotropy directions for the hardening (arguably identical to $\boldsymbol{W}^{A}$ since they are also due to the same texture evolution) and

$\boldsymbol{B}_{w}:=\boldsymbol{E}^{p e} \boldsymbol{B}-\boldsymbol{B} \boldsymbol{E}^{p e}$

Then, from Eqs. (34) and (39), the Dissipation $\dot{\mathcal{D}}=\mathcal{P}-\dot{\psi}$ is
$\mathbb{M}_{\dot{\boldsymbol{E}}}^{\boldsymbol{D}}=\sum_{i=1}^{3}\left(\lambda_{i}^{e}\right)^{2} \boldsymbol{M}_{i} \otimes \boldsymbol{M}_{i}+\sum_{i=1}^{3} \sum_{j \neq i} \frac{1}{2} \frac{\left(\lambda_{j}^{e}\right)^{2}-\left(\lambda_{i}^{e}\right)^{2}}{\ln \lambda_{j}^{e}-\ln \lambda_{i}^{e}} \boldsymbol{M}_{j} \stackrel{s}{\otimes} \boldsymbol{M}_{i}$

where $\lambda_{i}^{e}$ and $\boldsymbol{N}_{i}$ are the eigenvalues and eigenvectors of the elastic strains, $\boldsymbol{M}_{i}:=\boldsymbol{N}_{i} \otimes \boldsymbol{N}_{i}$ are the structural tensors and we defined the fourth order tensors with both minor and major symmetries

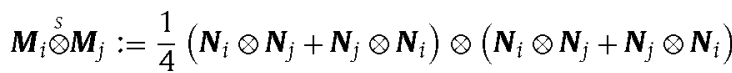

Hence

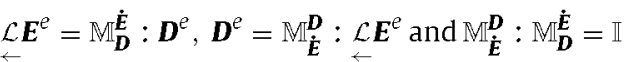

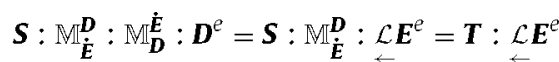

Then

$\boldsymbol{T}=\boldsymbol{S}: \mathbb{M}_{\dot{\boldsymbol{E}}}^{\boldsymbol{D}} \quad$ and $\quad \boldsymbol{S}=\boldsymbol{T}: \mathbb{M}_{\boldsymbol{D}}^{\dot{\boldsymbol{E}}}$

The Mandel stress tensor is

$\boldsymbol{\Xi}=\boldsymbol{C}^{e} \boldsymbol{S}=\boldsymbol{C}^{e}\left(\boldsymbol{T}: \mathbb{M}_{\boldsymbol{D}}^{\dot{\boldsymbol{E}}}\right)=\boldsymbol{T}:\left(\mathbb{S}^{M}+\mathbb{W}^{M}\right)=\boldsymbol{\Xi}_{s}+\boldsymbol{\Xi}_{w}$

where $\boldsymbol{\Xi}_{s}$ and $\boldsymbol{\Xi}_{w}$ are the symmetric and skew parts of $\boldsymbol{\Xi}$ and after some algebra we obtain (see details in Caminero et al. (2011))

$\mathbb{S}^{M}=\sum_{i=1}^{3} \boldsymbol{M}_{i} \otimes \boldsymbol{M}_{i}+\sum_{i=1}^{3} \sum_{j \neq i} \frac{\left(\lambda_{j}^{e}\right)^{2}+\left(\lambda_{i}^{e}\right)^{2}}{\left(\lambda_{j}^{e}\right)^{2}-\left(\lambda_{i}^{e}\right)^{2}}\left(\ln \lambda_{j}^{e}-\ln \lambda_{i}^{e}\right) \boldsymbol{M}_{j} \stackrel{s}{\otimes} \boldsymbol{M}_{i}$

with major and minor symmetries and

$\mathbb{W}^{M}=\sum_{i=1}^{3} \sum_{j \neq i}\left(\ln \lambda_{i}^{e}-\ln \lambda_{j}^{e}\right) \boldsymbol{M}_{i} \stackrel{s W}{\otimes M_{j}}$

with

$\boldsymbol{M}_{i} \stackrel{s W}{\otimes} \boldsymbol{M}_{j}:=\frac{1}{4}\left(\boldsymbol{N}_{i} \otimes \boldsymbol{N}_{j}+\boldsymbol{N}_{j} \otimes \boldsymbol{N}_{i}\right) \otimes\left(\boldsymbol{N}_{i} \otimes \boldsymbol{N}_{j}-\boldsymbol{N}_{j} \otimes \boldsymbol{N}_{i}\right)$

$$
\dot{\mathcal{D}}=\boldsymbol{S}: \boldsymbol{D}^{e}+\boldsymbol{\Xi}_{s}: \boldsymbol{D}^{p}+\boldsymbol{\Xi}_{w}: \boldsymbol{W}^{p}+\underbrace{\beta^{p e}: \boldsymbol{D}^{p e}+\boldsymbol{\beta}^{p d}: \boldsymbol{D}^{p d}}_{=0}+\underbrace{\boldsymbol{T}_{w}^{p e}: \boldsymbol{W}^{p e}+\boldsymbol{T}_{w}^{p d}: \boldsymbol{W}^{p d}}_{=0}
$$$$
-\boldsymbol{T}: \mathcal{L} \boldsymbol{E}^{e}-\boldsymbol{T}_{w}: \boldsymbol{W}^{A}-\boldsymbol{B}: \mathcal{L} \boldsymbol{E}^{p e}-\boldsymbol{B}_{w}: \boldsymbol{W}^{H}
$$

An identification of terms which yields the correct meaning for the energetic plasticspin and energetic plastic deformation rate tensors (i.e. they produce no plastic dissipation) is

$\boldsymbol{W}^{A}=\boldsymbol{W}^{\text {pe }} \quad$ and $\quad \boldsymbol{D}^{\text {pe }}=\mathcal{L} \boldsymbol{E}^{\text {pe }}$

and then

$\boldsymbol{T}_{w}^{p e} \equiv \boldsymbol{T}_{w} \quad$ and $\quad \boldsymbol{\beta}^{p e} \equiv \boldsymbol{B}$

whereas no elastic dissipation in purely elastic deformations yields

$\boldsymbol{S}: \boldsymbol{D}^{e}=\boldsymbol{T}: \underset{\mathcal{L} \boldsymbol{E}^{e}}{ }$

Obviously, since both $\mathcal{L} \boldsymbol{E}^{e}$ and $\boldsymbol{D}^{e}$ are measures of the elastic strain rates, they can be related to each other. As it can be easily checked, this relation is given by the fourth order tensors

$\mathbb{M}_{\boldsymbol{D}}^{\dot{\boldsymbol{E}}}=\sum_{i=1}^{3} \frac{1}{\left(\lambda_{i}^{e}\right)^{2}} \boldsymbol{M}_{i} \otimes \boldsymbol{M}_{i}+\sum_{i=1}^{3} \sum_{j \neq i} 2 \frac{\ln \lambda_{j}^{e}-\ln \lambda_{i}^{e}}{\left(\lambda_{j}^{e}\right)^{2}-\left(\lambda_{i}^{e}\right)^{2}} \boldsymbol{M}_{j} \stackrel{s}{\otimes} \boldsymbol{M}_{i}$ $\overline{\text { For moderately large elastic stretches } \lambda_{i}^{e} \text { (an hypothesis typically }}$ employed in computational elastoplasticity) $\mathbb{S}^{M} \simeq \mathbb{I}$ so one obtains $\boldsymbol{\Xi}_{s} \simeq \boldsymbol{T}$. It is straightforward to also verify that (this holds without any hypothesis)

$\boldsymbol{T}: \mathbb{W}^{M}=\boldsymbol{E}^{e} \boldsymbol{T}-\boldsymbol{T E}^{e}=: \boldsymbol{T}_{w}=\boldsymbol{\Xi}_{w}$

so for practical purposes both stress tensors $\boldsymbol{\Xi}_{s}$ and $\boldsymbol{T}$ and both tensors $\boldsymbol{\Xi}_{w}$ and $\boldsymbol{T}_{w}$ have the same meaning and the same physical interpretation. For large elastic strains, simply $\boldsymbol{\Xi}_{s}=\boldsymbol{T}: \mathbb{S}^{M}$ and $\boldsymbol{T}_{w}=$ $\boldsymbol{\Xi}_{w}$.

If the tensor $\mathbb{H}$ is isotropic (another common choice), then $\boldsymbol{B}_{w}=$ $\mathbf{0}$ and the value of $\mathbf{W}^{H}$ is irrelevant, otherwise a logical choice would be $\boldsymbol{W}^{A}$. Then

$$
\begin{aligned}
\dot{\mathcal{D}}^{p} & =\boldsymbol{\Xi}_{s}: \boldsymbol{D}^{p}+\boldsymbol{\Xi}_{w}: \boldsymbol{W}^{p}+\boldsymbol{\beta}^{p d}: \boldsymbol{D}^{p d}+\boldsymbol{T}_{w}^{p d}: \boldsymbol{W}^{p d} \\
& =\boldsymbol{\Xi}_{s}: \boldsymbol{D}^{p}+\boldsymbol{\Xi}_{w}: \boldsymbol{W}^{p}-\boldsymbol{B}: \boldsymbol{D}^{p e}-\boldsymbol{T}_{w}: \boldsymbol{W}^{A}
\end{aligned}
$$

We now consider three yield functions of the type $f_{y}\left(\boldsymbol{\Xi}_{s}-\boldsymbol{B}, \kappa\right)$, $g_{y}\left(\boldsymbol{B}, \kappa^{B}\right)$ and $h_{y}\left(\boldsymbol{\Xi}_{w}, \kappa^{w}\right)$. The augmented Lagrangian using the multipliers $\dot{t}, \dot{\gamma}, \dot{\lambda}$, is

$\mathcal{L}=\boldsymbol{\Xi}_{s}: \boldsymbol{D}^{p}+\boldsymbol{\Xi}_{w}: \boldsymbol{W}^{p}-\boldsymbol{B}: \boldsymbol{D}^{p e}-\boldsymbol{T}_{w}: \boldsymbol{W}^{A}-\dot{t} f_{y}-\dot{\gamma} g_{y}-\dot{\lambda} h_{y}$ 
and the principle of maximum dissipation yields

$\nabla \mathcal{L}=0 \Rightarrow\left\{\begin{array}{l}\boldsymbol{D}^{p}=\dot{t} \partial f_{y} / \partial \boldsymbol{\Xi}_{s} \\ \boldsymbol{W}^{p d}=\boldsymbol{W}^{p}-\boldsymbol{W}^{A}=\dot{\lambda} \partial h_{y} / \partial \boldsymbol{\Xi}_{w} \\ \boldsymbol{D}^{p e}=\dot{t} \partial f_{y} / \partial \boldsymbol{\Xi}_{s}-\dot{\gamma} \partial g_{y} / \partial \boldsymbol{B}=\boldsymbol{D}^{p}-\boldsymbol{D}^{p d}\end{array}\right.$

with the proper Kuhn-Tucker and consistency conditions.

The tensor $\mathbf{W}^{p d}$ was already identified in Montáns and Bathe (2007) as the dissipative plastic spin, although not derived from the current energetic/dissipative framework. The tensor $\boldsymbol{W}^{A}$ was therein explicitly set to modify the stored energy function, but this theory brings a more clear meaning to it. The first Equation of (62) is the usual flow rule. The second Equation of (62) gives the associative flow rule for the dissipative spin. This spin is associated to texture evolution, see for example (Kim et al., 2009). The third Equation of (62) is the hardening rule and it is a direct consequence of the usual assumption $f_{y}\left(\boldsymbol{\Xi}_{s}-\boldsymbol{B}, \kappa\right)$.

\subsection{The Armstrong and Frederick hardening rule}

The Armstrong and Frederick hardening rule may also be obtained at large strains with similar choices as in the small strains model. Using Eqs. (47) and $\left(40_{2}\right)$ we have

$\mathcal{L} \boldsymbol{B}=\mathcal{L} \boldsymbol{E}^{\text {pe }}: \mathbb{H}=\boldsymbol{D}^{\text {pe }}: \mathbb{H}$

Using the same hardening $\mathbb{H}=\frac{2}{3} H \mathbb{P}^{D}$ where $\mathbb{P}^{D}$ is the deviatoric projector tensor and $g_{y}=\frac{1}{2}\|\boldsymbol{B}\|^{2}$, from Eq. $\left(62_{3}\right)$

$\underset{\leftarrow}{\mathcal{L} B}=\frac{2}{3} H\left(\dot{t} \frac{\partial f_{y}}{\partial \boldsymbol{\Xi}_{s}}-\dot{\gamma} \boldsymbol{B}\right)$

so with the same viscoplastic-like relationship given in Eq. (11) we obtain the large strain version of the hardening rule

$$
\underset{\leftarrow}{\mathcal{L} B}=\dot{t}\left(\frac{2}{3} H \frac{\partial f_{y}}{\partial \boldsymbol{\Xi}_{s}}-b \boldsymbol{B}\right)
$$

\subsection{Texture evolution}

In References (Montáns and Bathe, 2007) and (Kim et al., 2009) we presented a model for texture evolution which predicts the experimental results of Kim and Yin (1997). This model is more clearly explained and motivated by the present theory, being the practical (and computational) result the same. In Montáns and Bathe (2007) we considered a linear relationship between the spins

$\boldsymbol{W}^{A}=\rho \boldsymbol{W}^{p}$

and

$\boldsymbol{W}^{p d}=(1-\rho) \boldsymbol{W}^{p}$

where $\rho$ is a material parameter with now the clear meaning of being the amount of the plastic spin which is energetic. Then, as for the case of the Armstrong-Frederick rule we consider a function for the skew part of the Mandel stress tensor in the form (with $\kappa^{w}=0$ )

$h_{y}=\frac{1}{2}\left\|\boldsymbol{\Xi}_{w}\right\|^{2}$

and a viscoplastic-like relationship between the effective plastic rotation rate and the effective plastic strain rate, which as with Eq. (11), can be written in terms of the consistency parameters (see details in Montáns and Bathe (2007)) as

$\dot{\lambda}=\left\langle\frac{h_{y}}{\omega}\right\rangle^{m} \dot{t}$ where $\varpi$ and $m$ are material parameters. Eq. (69) is motivated on the relation that exists in crystal plasticity between both contributions to the plastic flow. The model fits nicely in this theory, which enhances its physical interpretation.

\subsection{Multilinear kinematic hardening models}

Multilayer models (Montáns, 2001; Montáns and Caminero, 2007) may be cast in the present theory even at large strains. Simply consider the following split accounting for $N$ layers

$\boldsymbol{D}^{p e}=\sum_{i=1}^{N} \boldsymbol{D}_{i}^{p e}$

and the associated stresses $\boldsymbol{\beta}_{i}^{\text {pe }}$. Then proceed in a similar way as in the cited references, considering yield functions for the energetic stresses.

\section{Conclusions}

In this paper we presented a continuum mechanics theory for large strain anisotropic elastoplasticity capable of modeling mixed nonlinear kinematic-isotropic hardening and texture evolution. The theory is based on the Lee multiplicative decomposition of the deformation gradient into elastic and plastic parts. A decomposition motivated from Lion's decomposition of the plastic deformation gradient into energetic and dissipative contributions is introduced, but our proposal is to consider a decomposition in rate form. The resulting additive decomposition of the plastic deformation rate and plastic spin tensors yield meaningful contributions in the dissipation equation. A more clear physical interpretation of a formulation on texture evolution capable of predicting Kim and Yin experiments is obtained by the present theory and nonlinear kinematic hardening of the Armstrong and Frederick type or multilayer (Mroz-type) models may also be derived as special cases even in anisotropic elastoplasticity.

The formulation is developed using logarithmic stress and strain measures which may be used to define a quadratic stored energy function. If an exponential mapping is employed to integrate the plastic deformation gradients, these ingredients are known to allow for relatively simple and efficient incremental computational algorithms in which the plastic deformation is integrated using a small strains procedure and the large strain kinematics reduce to a geometric pre- and post-processor (Caminero et al., 2011).

\section{Acknowledgement}

Financial support has been obtained from projects DPI200805423 and DPI2011-26635 of the Ministerio de Ciencia e Innovación of Spain.

\section{References}

Anand, L., On, H., 1979. Hencky's approximate strain-energy function for moderate deformations. Journal of Applied Mechanics T-ASME 46, 78-82.

Bathe, K.J., Montáns, F.J., 2004. On modelling mixed hardening in computational plasticity. Computers and Structures 82, 535-539.

Bathe, K.J., 1996. Finite Element Procedures. Prentice-Hall, New Jersey.

Boheler, J.P., Koss, S., 1991. Evolution of anisot ropy in sheet steels subjected to offaxes large deformation. In: Brueler, O., Mannl, V., Najar, J. (Eds.), Advances in Continuum Mechanics. Springer, Berlin.

Caminero, M.A., Montáns, F.J., Bathe, K.J., 2011. Modeling large strain anisotropic elasto-plasticity with logarithmic strain and stress measures. Computers and Structures 89, 826-843.

Choi, Y., Walter, M.E., Lee, J.K., Han, C.S., 2006a. Observations of anisotropy evolution and identification of plastic spin parameters by uniaxial tensile tests. Journal of Mechanics of Materials and Structures 1, 303-324. 
Choi, Y., Han, C.S., Lee, J.K., Wagoner, R.H., 2006b. Modeling multiaxial deformation of planar anisotropic elasto-plastic materials part $i$ : Theory. International Journal of Plasticity 22, 1745-1764.

Dafalias, Y.F., 1985. The plastic spin. Journal of Applied Mechanics. Transactions of the ASME 52, 865-871.

Dafalias, Y.F., 1998. The plastic spin: necessity or redundancy? International Journal of Plasticity $14,909-931$.

Eterović, A.D., Bathe, K.J., 1990. A hyperelastic-based large strain elasto-plastic constitutive formulation with combined isotropic-kinematic hardening using the logarithmic stress and strain measures. International Journal for Numerical Methods in Engineering 30, 1099-1114.

Gurtin, M.E., Fried, E., Anand, L., 2010. The Mechanics and Thermodynamics of Continua. Cambridge University Press, New York.

Hennan, D.L, Anand, $L, 2009$. A large deformation theory for rate-dependent elastic-plastic materials with combined isotropic and kinematic hardening. International Journal of Plasticity 25, 1833-1878.

Kim, K.H., Yin, J.J., 1997. Evolution of anisotropy under plane stress. Journal of Mechanics and Physics of Solids 45, 841-851.

Kim, D.N., Montáns, F.J., Bathe, K.J., 2009. Insight into a model for large strain anisotropic elasto-plasticity. Computational Mechanics 44, 651-668.

Kojic, M., Bathe, K.J., 2005. Inelastic Analysis of Solids and Structures. SpringerVerlag, New York.

Kuroda, M., Tvergaard, V., 2001. Plastic spin associated with a non-normality theory of plasticity. European Journal of Mechanics A-Solids 20, 893-905.

Lemaitre, J., Chaboche, J.L., 1990. Mechanics of Solid Materials. Cambridge University Press, Cambridge.

Lion, A., 2000. Constitutive modelling in finite thermoviscoplasticity: a physical approach based on nonlinear rheological models. International Journal of Plasticity $16,469-494$

Loret, B., 1983. On the effects of plastic rotation in the finite deformation of anisotropic elastoplastic materials. Mechanics of Materials 2, 287-304.

Menzel, A., Steinmann, P., 2003. On the spatial formulation of anisotropic multiplicative elasto-plasticity. Computer Methods in Applied Mechanics and Engineering $105,151-180$.

Miehe, $C$, Apel, N., Lambrecht, M, 2002. Anisotropic additive plasticity in the logarithmic space: modular kinemat ic formulation and implementation based on incremental minimization principles for standard materials. Computer Methods in Applied Mechanics and Engineering 191, 5383-5426.
Montáns, F.J., Bathe, K.J., 2005. Computational issues in large st rain elasto-plasticity. An algorithm for mixed hardening and plastic spin. International Journal for Numerical Methods in Engineering 63, 159-196.

Montăns, F.J., Bathe, K.J., 2007. Towards a model for large strain anisotropic elastoplasticity. In: Onate, E., Owen, R. (Eds.), Chapter in Computational Plasticity. Springer-Verlag, pp. 13-36.

Montáns, FJ. Caminero, M.A, 2007. On the consistency of nested surfaces models and their kinematic hardening rules. International Journal of Solids and Struc tures $44,5027-5042$.

Montắns, F.J., 2001. Implicit multilayer $\mathrm{J}_{2}$-plasticity using Prager's transla tion rule. International Journal for Numerical Methods in Engineering 50 $347-375$

Montáns, F.J., 2004. Implicit plane stress algorithm for multilayer $\mathrm{J}_{2}$-plasticity using Prager-Ziegler translation rule. International Journal for Numerical Methods in Engineering 59, 409-418.

Papadopoulos, P., Lu, J., 2001. On the formulation and numerical solut ion of problems in anisotropic finite plasticity. Computer Methods in Applied Mechanics and Engineering 190, 4889-4910.

Schmidt, I., 2005. Some comments on formulations of anisotropic plasticity. Computational Materials Science $32,518-523$.

Simó, J.C., 1992. Algorithms for static and dynamic multiplicative plasticity that preserve the classical return mapping schemes of infinitesimal theory. Computer Methods in Applied Mechanics and Engineering 99, 61-112.

Tong, W., Tao, H., Jiang, X., 2004. Modeling the rotation of orthot ropic axes of sheet metals subjected to off-axis uniaxial tension. Journal of Applied Mechanics ASME $71,521-531$.

Truong, Q.H.K., Lippmann, H., 2001. Plastic spin and evolution of an anisotropic yield condition. International Journal of Mechanical Sciences 43, 1969-1983.

Ulz, M.H., 2009. A green-naghdi approach to finite anisotropic rate-independent and rate-dependent thermo-plasticity in logarithmic lagrangean strain-entropy space. Computer Methods in Applied Mechanics and Engineering 198 $3262-3277$.

Vladimirov, I.N., Pietryga, M.P., Reese, S., 2010. Anisotropic finite elastoplasticity with nonlinear kinematic and isotropic hardening and application to sheet metal forming. International Journal of Plasticity 26, 659-687.

Weber, G., Anand, L., 1990. Finite deformation constitutive equations and a time integration procedure for isotropic, hyperelastic-viscoplastic solids. Computer Methods in Applied Mechanics and Engineering 79, 173-202. 